\title{
Application of purified porcine collagen in patients with chronic refractory musculoskeletal pain
}

\author{
Hyunyoung Seong, Raing Kyu Kim, Youngjae Shin, Hye Won Lee, and Jae Chul Koh
}

Department of Anesthesiology and Pain Medicine, Korea University Anam Hospital, Seoul, Korea

Received April 27, 2020

Revised August 7, 2020

Accepted August 17, 2020

Handling Editor: Joon-Ho Lee

\section{Correspondence}

Jae Chul Koh

Department of Anesthesiology and

Pain Medicine, Korea University Anam

Hospital, 73 Goryeodae-ro, Seongbuk-gu,

Seoul 02841, Korea

Tel: +82-2-920-5632

Fax: +82-2-920-5630

E-mail: jaykoh@korea.ac.kr
Background: This study aimed to assess the potential efficacy of purified porcine atelocollagen (PAC) for the management of refractory chronic pain due to suspected connective tissue damage.

Methods: Patients treated with PAC were retrospectively evaluated. Patients with chronic refractory pain, suspected to have originated from musculoskeletal damage or defects with the evidence of imaging studies were included. Pain intensity, using the 11-point numerical rating scale (NRS), was assessed before the procedure, and 1 month after the last procedure.

Results: Eighty-eight patients were finally included for investigation. The mean NRS score was decreased from 5.8 to 4.1 after 1 month of PAC injection $(P<0.001)$. No independent factor was reported to be directly related to the decrease in NRS score by more than half.

Conclusions: Application of PAC may have potential as a treatment option for refractory chronic musculoskeletal pain. PAC might promote tissue recovery, act as a scaffold for repair, or directly reduce inflammation.

Key Words: Chronic Pain; Collagen; Connective Tissue; Injections; Musculoskeletal Pain; Pain Management; Procollagen; Regenerative Medicine.

\section{INTRODUCTION}

Musculoskeletal disorders are the most common sources of chronic pain worldwide $[1,2]$. These diseases result in functional disability and a heavy economic burden $[3,4]$. Due to its increasing prevalence and economic burden, the need for effective management of chronic musculoskeletal pain has grown.

Although arthritis and joint injuries of the tendons or ligaments can heal completely, chronic cumulative disorders can result in incomplete healing leading to chronic pain [5]. Several treatments have been introduced to restore the injury site by triggering a local inflammatory response in order to promote a normal healing cascade.
However, these treatments can result in problems that take months or even years for complete repair, prolonging the pain associated with local inflammatory reactions $[6,7]$. To compensate for this drawback, there has been increasing interest in application of collagen-which is the final product of the healing cascade-directly onto the injury site $[8,9]$.

Type I collagen is found in skin, tendon, vasculature, organs, and bone. Purified porcine atelocollagen (PAC) (Coltrix Tendoregen ${ }^{\mathrm{TM}}$; Ubiosis, Seongnam, Korea) is a soluble type I collagen with low immunogenicity, due to its lack of an antigenic telopeptide [10], and good biocompatibility [11]. PAC injection has been tried in various areas [12-14], but there are only a few studies that have investigated its (c) This is an open-access article distributed under the terms of the Creative Commons Attribution Non-Commercial License (http://creativecommons.org/licenses/by-nc/4.0/), which permits unrestricted non-commercial use, distribution, and reproduction in any medium, provided the original work is properly cited.

(C) The Korean Pain Society, 2020
Author contributions: Hyunyoung Seong: Data analysis, Writing/manuscript preparation; Raing Kyu Kim: Investigation, Data acquisition, Writing/ manuscript preparation; Youngjae Shin: Data acquisition, Writing/manuscript preparation; Hye Won Lee: Study conception, Data acquisition; Jae Chul Koh: Study conception, Data analysis, Writing/manuscript preparation. 
efficacy in the treatment of chronic pain. As such, the aim of this study was to assess the potential of PAC injection for management of refractory chronic pain in patients with suspected connective tissue damage.

\section{MATERIALS AND METHODS}

This retrospective medical record review study was approved by the Institutional Review Board of Korea University Anam Hospital (approval no. 2020AN0045) and have followed the principles outlined in the Declaration of Helsinki for all human or animal experimental investigations. Patient identification data were encoded and scrambled using a restricted computer to protect the privacy of all subjects.

The data were collected on patients (ages 30-90 yr) who visited the pain center for management of chronic pain lasting more than 3 months. Demographic data was collected at the first visit. At every visit, the pain intensity was evaluated using an 11-point numerical rating scale (NRS) $(0-10 ; 0=$ no pain/nausea, $10=$ worst imaginable pain $/$ nausea). Follow-ups occurred every 1-2 weeks, and any unexpected adverse events were recorded.

Patients who experienced chronic refractory pain, suspected to originate from damage or defects of ligaments, tendons, muscle, or biomembranes, were evaluated for eligibility. The inclusion criteria were confirmation of damage or defects at the site of pain, compared to the painless contralateral side using imaging studies, such as x-ray, computed tomography, magnetic resonance image, or ultrasound. Patients with damage or defects to the bone, muscle, joint, ligament, or tendon were included according to the following criteria. For skeletal defects, patients with chronic pain which might be associated with malunion or deformation were included. Pain originating from the muscle was diagnosed with a significant defect or rupture at the muscle fibers. Ligament- or tendon-associated pain was diagnosed with confirmation of damage to the integrity of the structures connecting the bone and muscle. In addition, patients with significant defects in the joint capsule, including degeneration or injury, were also included. Chronic refractory pain was defined as chronic pain that failed to decrease by more than a half despite one or more pain intervention procedures with conservative management. The exclusion criteria included lack of data for analysis and lack of follow-up before 1 month after treatment.

For the treatment of chronic pain, a mixture was prepared using equal amount of PAC and local anesthetics (total $2 \mathrm{~mL}$ ) to reduce pain during injection. After sterilization, the mixture was injected into the space between the surrounding tissue and the site of the greatest suspected musculoskeletal damage or defect that may have irritated adjacent structures, causing the chronic pain. The procedures were performed under ultrasound guidance. When the application site was large, the treatment was applied up to 3 times at intervals of 1 to 2 weeks, but only when the first procedure was effective.

Patients' demographic data and pre-procedural pain profiles-including age, sex, comorbidities (i.e., hypertension or diabetes mellitus), duration of pain, and presence of tenderness at the pain site-were evaluated. Patients' diagnosis was classified into 4 categories (i.e., skeletal, muscular, ligament and tendon, or joint) and recorded. The site of pain was categorized and recorded by region (i.e., spine, upper limb, lower limb, trunk, and other). Pain intensity, using the 11-point NRS, was assessed before the procedure, and 1 month after the last procedure.

\section{Statistical analysis}

Statistical analysis was performed using SAS software version 9.4 (SAS Institute, Cary, NC). Results were expressed as mean \pm standard deviation, or number of patients. We compared pre- and post-procedural NRS with a paired $t$ test or Wilcoxon signed-rank test for parametric and nonparametric data. Simple logistic regression analysis was used to identify factors associated with a decrease in pain intensity by more than half. Odds ratios (ORs) and $95 \%$ confidence intervals were estimated for each factor. $P$ values $<0.05$ were considered statistically significant.

\section{RESULTS}

A total of 135 patients were assessed for enrollment. Among them, 32 were excluded due to incomplete medical records, and 15 were excluded due to lack of follow-up. Of the initial 135, 88 patients were ultimately included in the study (Fig. 1). Demographic and pre-procedural data of the 88 analyzed patients are presented in Table 1 . The mean NRS score was 5.8 before the procedure. There were 31 , 13,25 , and 19 patients who were diagnosed in the skeletal, muscular, ligament and tendon, and joint category, re-

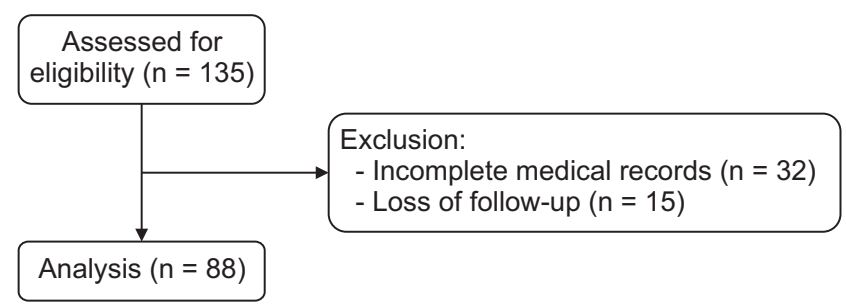

Fig. 1. Flow chart indicating patient selection and exclusion criteria. 
Table 1. Demographic and Pre-procedural Data

\begin{tabular}{lc}
\hline \multicolumn{1}{c}{ Variable } & Data $(\mathrm{n}=88)$ \\
\hline Age $(\mathrm{yr})$ & $68.2 \pm 12.6$ \\
Sex (M/F) & $26 / 62$ \\
Hypertension & 38 \\
Diabetes mellitus & 18 \\
Duration of pain (mo) & $28.9 \pm 35.2$ \\
Pre-procedural NRS (0-10) & $5.8 \pm 1.7$ \\
Tenderness at pain site & 37 \\
Categorical diagnosis & \\
Skeletal & 31 \\
Muscular & 13 \\
Ligament or tendon & 25 \\
Joint & 19 \\
Pain region & \\
Spine & 45 \\
Upper limb & 13 \\
Lower limb & 21 \\
Trunk & 4 \\
Other & 5
\end{tabular}

Values are presented as mean \pm standard deviation or number only. NRS: numerical rating scale.

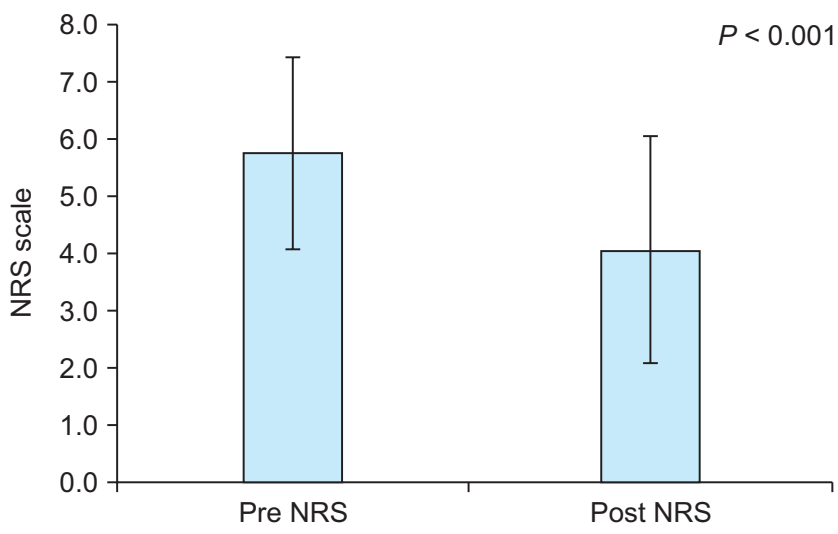

Fig. 2. Change in the numerical rating scale (NRS) score 1 month after porcine atelocollagen injection.

spectively. The most common injection site was the spine, followed by the lower limb, upper limb, and trunk.

One month after PAC injection, the mean NRS score had reduced to 4.1 ( $P<0.001$, Fig. 2$)$. Table 2 lists the various associated factors, and their respective number of patients whose NRS score decreased by more than half. There was no independent factor, including the diagnosis category and the site of pain, which was identified as being associated with a decrease in pain intensity by more than half. No adverse effects that might have been caused by treatment were recorded during the follow-up period.
Table 2. Factors Associated with Decrease in Pain Intensity by More Than a Half

\begin{tabular}{|c|c|c|c|}
\hline Predictor & Effect & $\begin{array}{c}\text { Odds ratio (95\% } \\
\text { confidence interval) }\end{array}$ & $P$ value \\
\hline Total & $37(42.0)$ & & \\
\hline \multicolumn{4}{|l|}{ Age (yr) } \\
\hline$<65$ & $16(47.1)$ & Ref & \\
\hline$\geq 65$ & $21(48.9)$ & $0.72(0.30-1.71)$ & 0.450 \\
\hline \multicolumn{4}{|l|}{ Sex } \\
\hline Male & $9(34.6)$ & Ref & \\
\hline Female & $28(45.2)$ & $1.56(0.60-4.02)$ & 0.362 \\
\hline Hypertension & $14(36.8)$ & $0.69(0.29-1.62)$ & 0.390 \\
\hline Diabetes mellitus & $8(44.4)$ & $1.13(0.40-3.21)$ & 0.817 \\
\hline \multicolumn{4}{|l|}{ No. of interventions } \\
\hline 1 & $3(30.0)$ & Ref & \\
\hline 2 & $7(43.8)$ & $1.81(0.34-9.68)$ & 0.486 \\
\hline$\geq 3$ & $27(43.5)$ & $1.80(0.43-7.62)$ & 0.425 \\
\hline \multicolumn{4}{|l|}{ Categorical diagnosis } \\
\hline Skeletal & $12(48.0)$ & Ref & \\
\hline Muscular & $6(33.3)$ & $0.54(0.15-1.90)$ & 0.339 \\
\hline Ligament or tendon & $11(47.8)$ & $0.99(0.32-3.09)$ & 0.990 \\
\hline Joint & $8(36.4)$ & $0.62(0.19-2.00)$ & 0.422 \\
\hline \multicolumn{4}{|l|}{ Tenderness at pain site } \\
\hline No & $16(40.0)$ & Ref & \\
\hline Yes & $21(43.8)$ & $1.17(0.50-2.73)$ & 0.723 \\
\hline \multicolumn{4}{|l|}{ Pain region } \\
\hline Spine & $17(37.8)$ & Ref & \\
\hline Upper limb & $3(23.1)$ & $0.49(0.12-2.05)$ & 0.332 \\
\hline Lower limb & $11(52.4)$ & $1.81(0.64-5.16)$ & 0.266 \\
\hline Trunk & $3(75.0)$ & $4.94(0.48-51.40)$ & 0.181 \\
\hline Other & $3(60.0)$ & $2.47(0.37-16.32)$ & 0.181 \\
\hline
\end{tabular}

Values are presented as number (\%) or number (95\% confidence interval).

Effect: number of patients whose pain intensity decreased by more than half, No. of intervention: number of previous intervention procedures before porcine atelocollagen.

\section{DISCUSSION}

This retrospective study evaluated the potential of PAC injection treatment in the management of refractory chronic pain due to suspected connective tissue damage. In the present study, we studied patients who were not responding to other conservative treatments. In these patients, normal recovery or inflammatory reactions are usually attenuated due to repeated damage $[15,16]$. For the treatment of patients with this condition, regenerative therapies such as prolotherapy have been introduced and developed. Despite application of various agents such as dextrose, polydeoxyribonucleotide or platelet rich plasma $[6,7,17,18]$, all these treatments aim to repair the damaged tissue by inducing an endogenous healing reaction via injection of a specific drug around the damaged structure, although the endogenous healing reaction may not occur or may be incomplete, especially owing to age [19]. Therefore, attempts 
have been made to directly inject collagen, the final product of the healing process, to treat chronic pain $[8,9]$.

PAC treatment has been used for regeneration of various organs, including bones [20], articular cartilages [14,21], and ligaments $[8,9]$. However, there are limited studies on the clinical application of PAC for chronic pain. To our best knowledge, this is the first study to evaluate the use of PAC for refractory chronic pain with suspected musculoskeletal damage. The significant reduction in the NRS score of patients treated in this study suggests the applicability of PAC injection for the treatment of intractable chronic pain caused by extensive tissue damage. In this study, we injected PAC into the space between the suspected damage or defect and its surrounding tissues. However, the exact mode of action or mechanism by which this application contributed to the improvement of the chronic pain is not fully known.

There are possible mechanisms which might explain the therapeutic effect of PAC. First, PAC may have directly promoted tissue recovery. Since collagen is the final product of tissue damage repair, it may have helped directly repair damaged tissue. Stopak et al. [22] reported that type I collagen injected into developing tissue was rearranged at the injection site. In addition, Suh et al. [8] reported histological and biomechanical repair at 12 weeks after fixation of PAC between damaged bone and tendon junctions. These studies may suggest the possibility that the healing cascade was promoted by direct engraftment of the end product. In the present study, the PAC that we administered around the damaged tissue may have been engrafted directly and was possibly involved in regeneration. However, such successful collagen engraftment may not be easily achieved through a simple injection into the degenerative tissue of elderly patients. Secondly, PAC might have acted as a scaffold for repair. This mechanism has been studied for bones, ligaments, tendons, and cartilage through laboratory studies $[8,12,14,20,21]$. PAC has a long half-life because of its resistance to degradation by enzymatic breakdown, due to its triple helix structure and nonimmunity $[14,23]$. Therefore, it is estimated that PAC might have acted as a scaffold to facilitate the tissue regeneration reaction around damaged tissue in the present study. In this respect, it is possible that using progenitor cells or proliferation-related factors may have a synergistic effect $[19,24,25]$. Finally, the effect might have been caused by the reduction of inflammation. Because PAC, which does not easily degrade, may simply remain around the injection site, and thus reduce chronic inflammation through a protective role from repetitive stimulation by degeneration or damaged tissue.

In studies involving prolotherapy, the prognoses were different depending on the region of pain [26]. However, according to the results of the present study, the region of pain was not a significant factor impacting the therapeutic effect of the PAC. Differences between the mechanisms of prolotherapy and PAC application may explain this result, but a lack of statistical power, due to insufficient sample size, may also be the cause. A well-designed study with a larger sample size is necessary for more clarification.

There are some limitations to this study. Since there was no control group, it was not possible to present a relative comparative analysis of the effects from other treatments. However, because this study targeted patients with refractory chronic pain-for whom there was no relief after other various treatments, including anti-inflammatory injection, prolotherapy, medication or physical therapy, et al.-it was difficult to designate specific treatments as a control. In addition, the area of pain targeted for this study is diverse, which has limitations in specifying the indications for the procedure. The short follow-up period of 1 month and a lack of control over the use of other treatments, due to the retrospective study design, were other limitations of this study. Nevertheless, this study is significant in that it is the first study to evaluate the possibility of PAC as a treatment option for chronic intractable musculoskeletal disorders. It is anticipated that further well-designed studies with histological investigation in the future will give a better understanding of the potential of PAC as a treatment option for chronic refractory pain.

In conclusion, NRS score reduction was obtained by administering PAC to patients with refractory chronic pain. Application of PAC may have potential as a treatment option for refractory chronic musculoskeletal pain.

\section{CONFLICT OF INTEREST}

No potential conflict of interest relevant to this article was reported.

\section{FUNDING}

No funding to declare.

\section{ORCID}

Hyunyoung Seong, https://orcid.org/0000-0001-5136-1058 Raing Kyu Kim, https://orcid.org/0000-0002-8030-819X Youngjae Shin, https://orcid.org/0000-0003-0946-3565 Hye Won Lee, https://orcid.org/0000-0003-3088-1921

Jae Chul Koh, https://orcid.org/0000-0002-1625-8650 


\section{REFERENCES}

1. Fayaz A, Croft P, Langford RM, Donaldson LJ, Jones GT. Prevalence of chronic pain in the UK: a systematic review and meta-analysis of population studies. BMJ Open 2016; 6: $\mathrm{e} 010364$

2. Velly AM, Mohit S. Epidemiology of pain and relation to psychiatric disorders. Prog Neuropsychopharmacol Biol Psychiatry 2018; 87(Pt B): 159-67.

3. Oh IH, Yoon SJ, Seo HY, Kim EJ, Kim YA. The economic burden of musculoskeletal disease in Korea: a cross sectional study. BMC Musculoskelet Disord 2011; 12: 157.

4. Yelin E, Weinstein S, King T. The burden of musculoskeletal diseases in the United States. Semin Arthritis Rheum 2016; 46: 259-60.

5. Killian ML, Cavinatto L, Galatz LM, Thomopoulos S. The role of mechanobiology in tendon healing. J Shoulder Elbow Surg 2012; 21: 228-37.

6. Hauser RA, Lackner JB, Steilen-Matias D, Harris DK. A systematic review of dextrose prolotherapy for chronic musculoskeletal pain. Clin Med Insights Arthritis Musculoskelet Disord 2016; 9: 139-59.

7. Squadrito F, Bitto A, Irrera N, Pizzino G, Pallio G, Minutoli L, et al. Pharmacological activity and clinical use of PDRN. Front Pharmacol 2017; 8: 224.

8. Suh DS, Lee JK, Yoo JC, Woo SH, Kim GR, Kim JW, et al. Atelocollagen enhances the healing of rotator cuff tendon in rabbit model. Am J Sports Med 2017; 45: 2019-27.

9. Kim M, Choi YS, You MW, Kim JS, Young KW. Sonoelastography in the evaluation of plantar fasciitis treatment: 3-month follow-up after collagen injection. Ultrasound Q 2016; 32: 327-32.

10. Hanai K, Kojima T, Ota M, Onodera J, Sawada N. Effects of atelocollagen formulation containing oligonucleotide on endothelial permeability. J Drug Deliv 2012; 2012: 245835.

11. Lynn AK, Yannas IV, Bonfield W. Antigenicity and immunogenicity of collagen. J Biomed Mater Res B Appl Biomater 2004; 71: 343-54.

12. Sheikh Z, Qureshi J, Alshahrani AM, Nassar H, Ikeda Y, Glogauer M, et al. Collagen based barrier membranes for periodontal guided bone regeneration applications. Odontology 2017; 105: 1-12.

13. Dunn MG, Tria AJ, Kato YP, Bechler JR, Ochner RS, Zawadsky
JP, et al. Anterior cruciate ligament reconstruction using a composite collagenous prosthesis. A biomechanical and histologic study in rabbits. Am J Sports Med 1992; 20: 507-15.

14. Lee HS, Oh KJ, Moon YW, In Y, Lee HJ, Kwon SY. Intra-articular injection of type I atelocollagen to alleviate knee pain: a double-blind, randomized controlled trial. Cartilage 2019 doi: 10.1177/1947603519865304.

15. Aström M, Rausing A. Chronic achilles tendinopathy. A survey of surgical and histopathologic findings. Clin Orthop Relat Res 1995; (316): 151-64.

16. Kane SF, Olewinski LH, Tamminga KS. Management of chronic tendon injuries. Am Fam Physician 2019; 100: 147-57.

17. Kim SH, Jeon YH, Park JW. Effects of prolotherapy on chronic musculoskeletal disease. Korean J Pain 2002; 15: 121-5.

18. Andia I, Abate M. Platelet-rich plasma: combinational treatment modalities for musculoskeletal conditions. Front Med 2018; 12: 139-52.

19. Clark D, Nakamura M, Miclau T, Marcucio R. Effects of aging on fracture healing. Curr Osteoporos Rep 2017; 15: 601-8.

20. Oliveira SM, Ringshia RA, Legeros RZ, Clark E, Yost MJ, Terracio $\mathrm{L}$, et al. An improved collagen scaffold for skeletal regeneration. J Biomed Mater Res A 2010; 94: 371-9.

21. Matsushita R, Nakasa T, Ishikawa M, Tsuyuguchi Y, Matsubara N, Miyaki S, et al. Repair of an osteochondral defect with minced cartilage embedded in atelocollagen gel: a rabbit model. Am J Sports Med 2019; 47: 2216-24

22. Stopak D, Wessells NK, Harris AK. Morphogenetic rearrangement of injected collagen in developing chicken limb buds. Proc Natl Acad Sci U S A 1985; 82: 2804-8.

23. Chang SW, Flynn BP, Ruberti JW, Buehler MJ. Molecular mechanism of force induced stabilization of collagen against enzymatic breakdown. Biomaterials 2012; 33: 3852-9.

24. Bayarsaikhan O, Kawai N, Mori H, Kinouchi N, Nikawa T, Tanaka E. Co-administration of myostatin-targeting siRNA and ActRIIB-Fc fusion protein increases masseter muscle mass and fiber size. J Nutr Sci Vitaminol (Tokyo) 2017; 63: 244-8.

25. Lee KI, Moon SH, Kim H, Kwon UH, Kim HJ, Park SN, et al. Tissue engineering of the intervertebral disc with cultured nucleus pulposus cells using atelocollagen scaffold and growth factors. Spine (Phila Pa 1976) 2012; 37: 452-8.

26. Covey CJ, Sineath MH Jr, Penta JF, Leggit JC. Prolotherapy: can it help your patient? J Fam Pract 2015; 64: 763-8. 